\title{
Importancia del subsidio de macroalgas sobre la abundancia y biología poblacional del anfípodo Orchestoidea tuberculata (Nicolet) en playas arenosas del centro sur de Chile
}

\author{
Importance of macroalgae subsidy on the abundance and population biology of the amphipod \\ Orchestoidea tuberculata (Nicolet) in sandy beaches of south central Chile
}

\author{
Cristian Duarte ${ }^{1}$, Eduardo Jaramillo ${ }^{2}$, Heraldo Contreras ${ }^{3}$, Karin Acuña ${ }^{1}$ y Jorge M. Navarro ${ }^{1}$ \\ ${ }^{1}$ Instituto de Biología Marina, Universidad Austral de Chile, casilla 567, Valdivia, Chile \\ ${ }^{2}$ Instituto de Ecología y Evolución, Universidad Austral de Chile, casilla 567,Valdivia, Chile \\ ${ }^{3}$ Instituto de Fomento Pesquero, Balmaceda 252, Puerto Montt, Chile \\ cduarte@uach.cl
}

\begin{abstract}
The talitrid amphipod Orchestoidea tuberculata (Nicolet) is one of the dominant species inhabiting the upper intertidal of a variety of morphodynamic types of exposed sandy beaches of the Chilean coast. The macroalgae stranded on the beach are the primary food source for this amphipod. In this study, we analyzed the influence of the availability of macroalgae and beach morphodynamic features on the population abundance and life history of $O$. tuberculata. Eleven beaches of the coast of Valdivia $\left(c a .39^{\circ} \mathrm{S}\right)$ were sampled, during a period of 13 months, to determine biomass of stranded macroalgae and beach characteristics. Seasonal faunal samples were collected to characterize the populations of $O$. tuberculata at each site. The results showed the configuration of two beach groups with significant differences in biomass of stranded macroalgae, independent of their morphodynamic features. The total abundance of amphipods, the abundance of females, ovigerous females, males and juveniles, as well as body size, were significantly higher at beaches with higher biomass of stranded macroalgae. Fecundity did not differ significantly between both beach groups, although the expected number of eggs per female was nine times higher at those beaches with the highest biomass of stranded macroalgae. These results suggest that food availability has a significant role on the population abundances and life history of $O$. tuberculata, independent of the morphodynamic type of sandy beaches, situation which departs from paradigmatic models in sandy beach ecology.
\end{abstract}

Key words: Morphodynamic state, trophic subsidy, stranded macroalgae

\section{Introducción}

Las playas arenosas expuestas del tipo micromareal (i.e. rango mareal inferior a $3 \mathrm{~m}$ ) son el hábitat costero más representado en las costas temperadas del Pacífico Oriental (Bascom 1980). El estado morfodinámico de este
Resumen.- El anfípodo talítrido Orchestoidea tuberculata (Nicolet) es una de las especies dominantes en el intermareal superior de variados tipos morfodinámicos de playas arenosas expuestas de la costa de Chile. Las macroalgas varadas sobre la playa son la fuente primaria de alimento para este anfípodo. En este estudio evaluamos la influencia de la disponibilidad de macroalgas y características morfodinámicas de las playas sobre las abundancias poblacionales e historia de vida de $O$. tuberculata. Se muestrearon 11 playas de la costa de Valdivia (ca. $39^{\circ} \mathrm{S}$ ), durante un periodo de 13 meses, para determinar la biomasa de algas varadas y las características de las playas. En cada sitio se recolectaron muestras estacionales para caracterizar las poblaciones de $O$. tuberculata. Los resultados mostraron la formación de dos grupos de playas con diferencias significativas en la biomasa de algas varadas, independiente de sus características morfodinámicas. La abundancia total de anfípodos, abundancia de hembras, hembras ovígeras, machos y juveniles, como también tamaño corporal fueron significativamente mayores en playas con mayor cantidad de macroalgas varadas. La fecundidad no difirió significativamente entre ambos grupos de playas, aunque el número esperado de huevos por hembra fue nueve veces mayor en playas con mayor cantidad de macroalgas. Estos resultados sugieren que la disponibilidad de alimento, tiene un rol significativo sobre las abundancias poblacionales e historias de vida de O. tuberculata, independiente del tipo morfodinámico de playas, situación que se aparta de modelos paradigmáticos en ecología de playas arenosas.

Palabras clave: Estado morfodinámico, subsidios tróficos, macroalgas varadas

tipo de playas es el resultado de la interacción entre el tamaño del grano de arena y las características de la ola, lo que se integra en el parámetro $\Omega$ o parámetro de Dean, cuyos valores permiten diferenciar playas reflectivas, intermedias y disipativas (Short 1996, 2006). Las primeras se caracterizan por presentar grano grueso, 
pendiente muy inclinada y olas de baja energía (valores de $\Omega \leq 1$ ), en tanto que las últimas por presentar arenas finas, pendientes suaves y olas de gran energía (valores de $\Omega>6$ ).

Varios estudios publicados durante los últimos años, sugieren que el estado morfodinámico de las playas influye de manera significativa sobre la riqueza de especies, abundancia y biomasa de la macrofauna intermareal que habita en estos hábitats costeros (Brown \& McLachlan 1990, Jaramillo \& McLachlan 1993, McLachlan \& Jaramillo 1995, McLachlan et al. 1993, 1996, 1998, Defeo et al. 2003), lo que ha generado la formulación de diferentes hipótesis tendientes a explicar el comportamiento de la macrofauna frente a distintas características morfodinámicas. La hipótesis autoecológica (McLachlan 1990), predice que la comunidad se estructura de acuerdo a las respuestas individuales de cada población a las características físicas del medioambiente. A su vez, la hipótesis de exclusión por efecto del barrido de la ola o 'swash' (McLachlan et al. 1993) propone un incremento en la riqueza, abundancia y biomasa de la macrofauna desde playas reflectivas a disipativas ( $c f$. McArdle \& McLachlan 1991, 1992). Por otra parte, la hipótesis de rigurosidad del hábitat (Defeo et al. 2003) combina las hipótesis anteriores (aplicadas a nivel comunitario) prediciendo que también las características de historia de vida (e.g. crecimiento, fecundidad) de las especies que viven en la zona de barrido de la ola incrementan desde playas reflectivas a disipativas. Recientemente, Defeo \& Gómez (2005) propusieron la hipótesis de protección del hábitat (aplicable a nivel poblacional), la cual predice que las especies supralitorales incrementan sus atributos poblacionales (e.g. abundancia total, abundancia de juveniles) hacia playas reflectivas, en oposición a las predicciones de las hipótesis planteadas anteriormente.

A pesar de la evidencia mostrada en estudios recientes, la relación existente entre la macrofauna y el estado morfodinámico de las playas, no siempre es consistente y ha mostrado variar considerablemente (e.g. Jaramillo et al. 2000, 2001, Dugan et al. 2003). A nivel comunitario, Jaramillo et al. (2000) encontraron que en seis playas del centro sur de Chile (Isla Grande de Chiloé), no ocurre un incremento en el número de especies y abundancia de macrofauna desde playas reflectivas a playas disipativas, como había sido predicho a partir de trabajos previos (e.g. Jaramillo \& McLachlan 1993, McLachlan \& Jaramillo 1995, McLachlan et al. 1993, 1996, 1998, Defeo et al. 2003). A nivel poblacional, las respuestas de la macrofauna frente al estado morfodinámico son aun más disímiles. Por ejemplo, Defeo et al. (1997) encontraron que la variabilidad espacial en la abundancia y tamaño corporal del isópodo cirolánido Excirolana braziliensis, no puede ser explicada por el estado morfodinámico de las playas, en tanto que Jaramillo \& McLachlan (1993) encontraron que ese mismo isópodo incrementó significativamente sus abundancias hacia playas con características disipativas. De esos trabajos se desprende que son especialmente los organismos que habitan en los niveles superiores de la playa (los cuales presentan una menor interacción con la zona de barrido de las olas), los que no responden consistentemente al estado morfodinámico de estas. Koop \& Field (1980) sugirieron que la macrofauna supralitoral estaría más afectada por la disponibilidad de alimento que por factores físicos.

Durante los últimos años, se ha comenzado a reconocer que los ecosistemas son, en muchos casos, abiertos e inter-relacionados y que patrones de escala local pueden estar fuertemente influenciados por factores externos al sistema (Lewin 1986, Polis et al. 1997, Rodríguez 2000, Nielsen \& Navarrete 2004). Entre estos factores, el flujo de alimento desde un sistema a otro (subsidio trófico) ha sido considerado como uno de los más importantes (Fairweather \& Quinn 1992, Bustamante et al. 1995, Bustamante \& Branch 1996, Polis et al. 1997). Los subsidios tróficos son especialmente importantes en playas arenosas expuestas, ya que debido a la baja productividad primaria de las mismas (Brown \& McLachlan 1990), la macrofauna que allí habita depende casi exclusivamente del arribo de alimento desde otros sistemas (Griffiths et al. 1983, Colombini et al. 2000). En muchas regiones temperadas, las macroalgas a la deriva provenientes de praderas algales o costas rocosas adyacentes (Rodríguez 2000, Rossi \& Underwood 2002, Dugan et al. 2003) son uno de los principales subsidios tróficos que arriban a la playa, representando el principal ítem alimentario para organismos típicos de los niveles superiores del intermareal, como anfípodos talítridos, isópodos oniscoídeos e insectos tenebriónidos y estafilínidos (Griffiths \& Stenton-Dozey 1981, Griffiths et al. 1983, Inglis 1989, Brown \& McLachlan 1990, Dugan et al. 2003). En otros sistemas costeros, el subsidio de macroalgas ha mostrado jugar un rol significativo en la organización de las poblaciones y comunidades (e.g. Bustamante et al. 1995, Polis et al. 1995, 1997). Sin embargo, en playas arenosas expuestas, donde existe una alta probabilidad que la disponibilidad de las macroalgas influya significativamente sobre la macrofauna que habita en los niveles superiores del intermareal, la respuesta de la macrofauna al subsidio de macroalgas ha recibido relativamente poca atención, enfatizándose en su lugar el rol de los factores físicos (e.g. McLachlan \& Jaramillo 1995, Jaramillo et al. 2000, Defeo \& Gómez 2005).

El anfípodo talítrido Orchestoidea tuberculata (Nicolet), es una de las especies numéricamente dominante en los niveles superiores de la zona intermareal 
de playas arenosas expuestas del litoral chileno, ubicadas entre $30^{\circ} \mathrm{S}$ y $42^{\circ} \mathrm{S}$ (Varela 1983, Jaramillo et al. 2000, 2003), pudiendo habitar en un amplio espectro de estados morfodinámicos de playa (Jaramillo et al. 2000). Los trabajos realizados hasta la fecha sobre $O$. tuberculata se han enfocado principalmente a explicar las abundancias poblacionales y rasgos de historia de vida de este anfípodo en relación las características físicas de las playas (e.g. Contreras et al. 2003, Jaramillo \& McLachlan 1993); sin embargo, los resultados obtenidos han sido altamente disímiles, mostrando en algunos casos incrementos y en otros disminuciones de sus atributos poblaciones desde playas reflectivas a playas disipativas. Ya que $O$. tuberculata depende casi exclusivamente del subsidio de macroalgas como fuente de alimento (Duarte 1974), en este estudio se plantea la hipótesis que las poblaciones de $O$. tuberculata están influenciadas por la disponibilidad de macroalgas varadas en la zona intermareal de la playa. Esta hipótesis predice que este anfípodo presenta los valores más altos de abundancia, tamaño corporal, éxito reproductivo y fecundidad en playas que presenten mayor cantidad de macroalgas varadas, independiente de las características físicas de las mismas. Para evaluar estas predicciones, se comparó la abundancia y los rasgos de historia de vida de $O$. tuberculata proveniente de once playas arenosas de la costa centro sur de Chile ( $c a .39^{\circ} \mathrm{S}$ ), con diferentes características físicas y cantidad de macroalgas varadas.

\section{Material y métodos}

\section{Área de estudio}

Los estudios de terreno se realizaron en 11 playas arenosas de la costa de Valdivia (centro sur de Chile; $c a$. $39^{\circ} \mathrm{S}$ ) (Fig. 1): San Ignacio (SI), La Misión (LM), Calfuco Sur (Ca S), Calfuco Norte $(\mathrm{Ca} \mathrm{N})$, Curiñanco Sur $(\mathrm{Cu}$ $\mathrm{S})$, Curiñanco Norte $(\mathrm{Cu} \mathrm{N})$, Caleta Curiñanco (CC), Mehuín (Me), Universitaria (Un), Pichicullín (Pi) y Cheuque (Che).
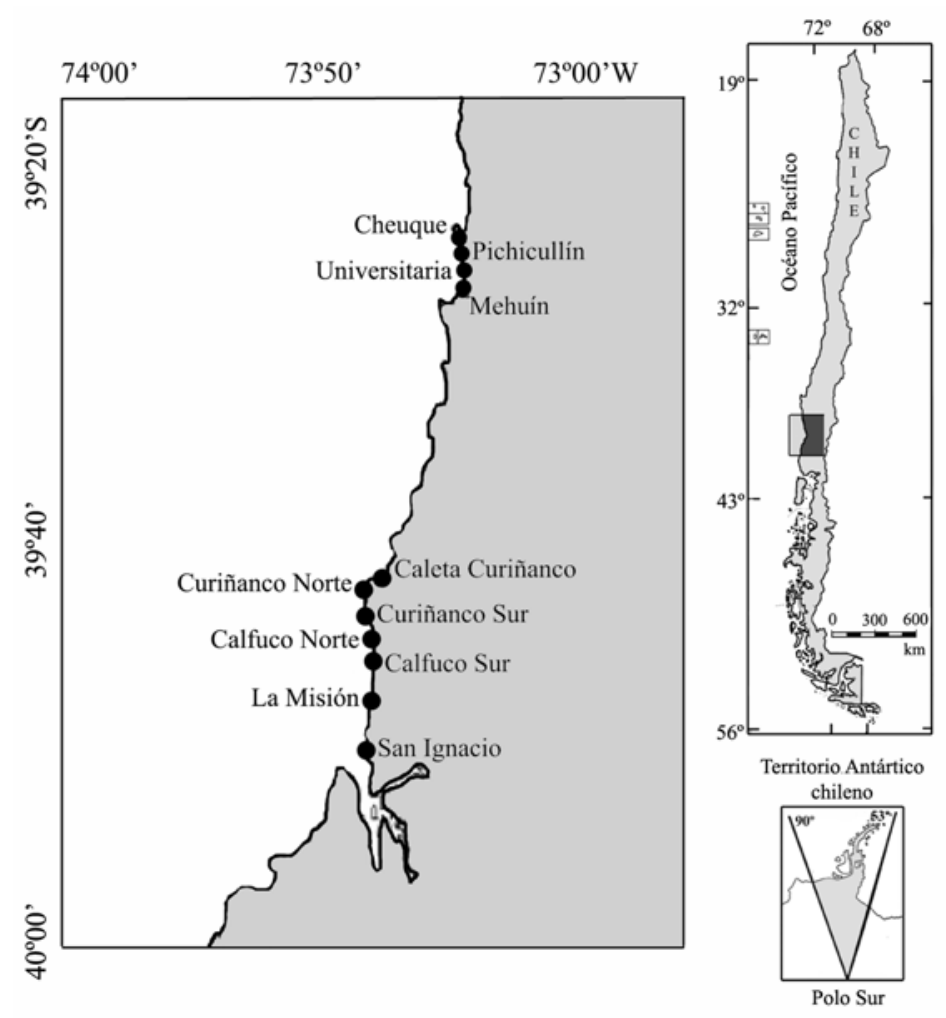

Figura 1

Ubicación geográfica de las playas estudiadas en la costa centro sur de Chile: San Ignacio (SI), La Misión (LM), Calfuco Sur (Ca S), Calfuco Norte (Ca N), Curiñanco Sur (Cu S), Curiñanco Norte (Cu N), Caleta Curiñanco (CC), Mehuín (Me), Universitaria (Un), Pichicullín (Pi) y Cheuque (Che)

Geographic location of the beaches studied on the coast of south central Chile: San Ignacio (SI), La Misión (LM), Calfuco Sur (Ca S), Calfuco Norte (Ca N), Curiñanco Sur (Cu S), Curiñanco Norte $(\mathrm{Cu} \mathrm{N})$, Caleta Curiñanco (CC), Mehuín (Me), Universitaria (Un), Pichicullín (Pi) and Cheuque (Che) 


\section{Cantidad de macroalgas varadas}

Durante las mareas bajas de sicigia del período mayo 2003-mayo 2004, se determinó mensualmente la cantidad (biomasa) de macroalgas varadas en cada playa. Se muestreó en cuatro transectos de $10 \mathrm{~m}$ de ancho cada uno, ubicados a lo largo de cada playa, separados equidistantes entre sí y extendidos a lo ancho de la zona intermareal. La distancia entre cada uno de esos transectos dependió del largo total de cada playa. Todas las macroalgas presentes en cada transecto fueron pesadas en terreno con una balanza de mano. Una vez pesadas, las algas fueron retiradas de la playa y depositadas sobre las dunas. Los datos fueron expresados en $\mathrm{kg} \mathrm{m}^{-1}$ de peso fresco.

\section{Características físicas de las playas}

Las características físicas de cada playa fueron analizadas mensualmente desde mayo 2003 hasta mayo 2004. En cada uno de los transectos donde se estimó la cantidad de macroalgas varadas, se determinó la inclinación de la zona intermareal mediante la técnica de Emery (1961). Se obtuvieron muestras superficiales de sedimento desde la zona de barrido de la ola, para análisis granulométricos (Emery 1938). Se estimó la altura de la ola $(\mathrm{n}=15)$, con una regla graduada en centímetros y alineada con el horizonte. El período de la ola, considerado como el tiempo transcurrido (segundos) entre dos olas que rompen, fue medido $(\mathrm{n}=15)$ con un cronómetro digital. Posteriormente, se calculó el parámetro $\Omega$ o parámetro de Dean mediante la siguiente formula: $\Omega=\mathrm{A} / \mathrm{Vs} * \mathrm{t}$, donde A es la altura de la ola $(\mathrm{cm})$, Vs es la velocidad de sedimentación de las partículas de arena $\left(\mathrm{cm} \mathrm{s}^{-1}\right)$ y t es el período de la ola (s). La velocidad de sedimentación de las partículas fue tomada de las tablas de Gibbs et al. (1971) sobre la base del tamaño medio de la partícula calculada de acuerdo al método computacional de momentos (Seward-Thompson \& Hails 1973). Como fue mencionado anteriormente, valores de $\Omega \leq 1$ corresponden a playas reflectivas, valores superiores a 1 y hasta 5 definen las playas intermedias, a la vez que valores de $\Omega$ superiores a 6 corresponden a playas disipativas (c.f. Short 1996, 2006).

\section{Abundancia y rasgos de historia de vida de Orchestoidea tuberculata}

En cada playa se recolectaron muestras estacionales (agosto 2003-abril 2004) para análisis faunísticos. Se muestreó sobre cuatro transectos perpendiculares a la línea de la costa, los cuales fueron coincidentes con aquellos donde se estimó la biomasa de macroalgas. Cada transecto se extendió a lo ancho de la distribución intermareal de los individuos enterrados de $O$. tuberculata. El ancho de la distribución intermareal de este anfípodo fue determinado de manera previa a cada muestreo. Para ello se obtuvieron muestras cualitativas con un cilindro plástico de $0,05 \mathrm{~m}^{2}$ de área, a intervalos regulares de $50 \mathrm{~cm}$ y hasta constatar la ausencia de anfípodos. Una vez determinado el ancho aproximado de la banda de distribución de $O$. tuberculata, se distribuyeron seis estaciones a intervalos regulares en cada transecto ( $c f$. Defeo \& Rueda 2002). En cada estación se recolectó una muestra de sedimento con un cilindro plástico de $0,05 \mathrm{~m}^{2}$ de área, enterrado a una profundidad de $30 \mathrm{~cm}$. Cuando una estación de muestreo coincidió con un depósito de algas, el core fue enterrado a través de ese depósito, retirándose posteriormente los restos de algas. La arena recolectada se filtró en cernidores con mallas de $1 \mathrm{~mm}$ de abertura. Los individuos retenidos fueron contados, sexados y medidos. La longitud corporal fue la distancia entre el extremo distal del rostrum y la base del telson, medida con una exactitud de 0,05 mm. Los anfípodos fueron clasificados en juveniles y adultos. Los juveniles fueron aquellos individuos de talla menor a $7 \mathrm{~mm}$ y sin caracteres sexuales secundarios reconocibles ( $c f$. Jaramillo et al. 1980). Los individuos adultos fueron categorizados como machos, hembras y hembras ovígeras.

\section{Fecundidad}

Para estimar la fecundidad de O. tuberculata se recolectaron hembras ovígeras en cada playa, las que fueron guardadas individualmente en tubos Eppendorf. Estas muestras se recolectaron durante octubre y noviembre de 2003, cuando se registran las mayores abundancias de hembras ovígeras de esta especie en la costa del centro sur de Chile (Jaramillo 1987). Posteriormente, las muestras se llevaron al laboratorio donde se midió la longitud corporal (ver arriba) y se contaron los estadios intramarsupiales (huevos y embriones) de las mismas. Luego, se realizó un ajuste lineal entre el número de estadios intramarsupiales y la talla de las hembras ovígeras.

\section{Análisis de datos}

Los valores de la biomasa de las macroalgas varadas y de las variables físicas de las playas durante todos los meses de muestreo fueron promediados para cada réplica (i.e. para cada transecto), acorde a Schoeman \& Richardson (2002) y Defeo \& Gómez (2005). Se utilizó esta aproximación analítica ya que permitió dar cuenta de la variabilidad intra-playa de las variables analizadas y a que el principal desafío de este análisis no fue detectar diferencias significativas entre distintos períodos de tiempo. 


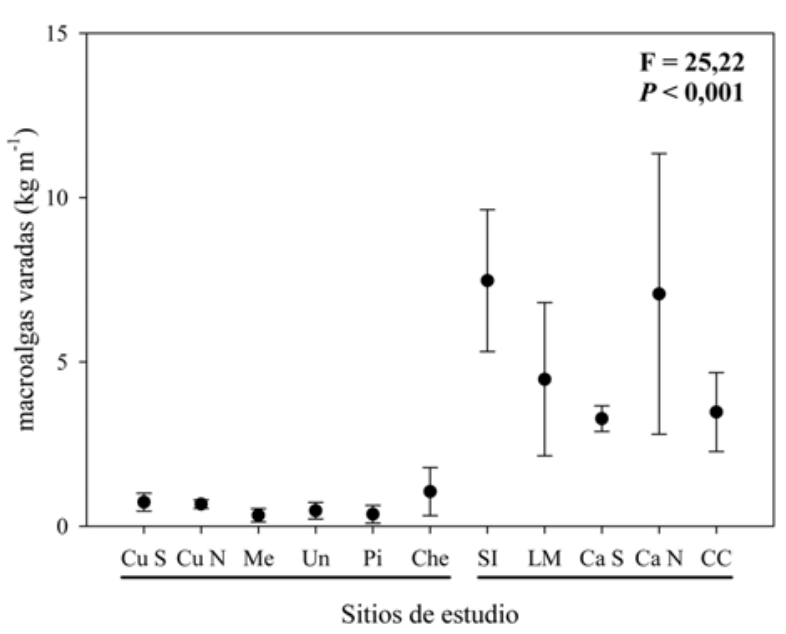

Figura 2

Biomasa de macroalgas varadas ( $\mathrm{kg} \mathrm{m}^{-1}$ peso húmedo) en las playas estudiadas. Los valores son promedios $( \pm 1$ desviación estándar) basados en cuatro transectos muestreados mensualmente $(n=13)$ (ver Material y métodos para detalles). Valores de $\mathrm{F}$ y $\boldsymbol{P}$ son resultantes del ANDEVA de una vía. Las líneas unen promedios que no difieren significativamente entre sí. Ver Fig. 1 para el nombre de las playas

Biomass of the stranded macroalgae $\left(\mathrm{kg} \mathrm{m}^{-1}\right.$ wt weight) on the beaches studied. The values are means $( \pm 1$ standard deviation) based on four transects sampled monthly $(\mathrm{n}=13)$ (see Material and methods for details). Values of $\mathrm{F}$ and $P$ are resulting from one-way ANOVA. Lines link means not significantly different among each others. See Fig. 1 for names of the beaches

Para comparar la biomasa promedio total (basado en cuatro transectos muestreados durante 13 meses consecutivos) de las macroalgas varadas entre las distintas playas, se llevaron a cabo análisis de varianza de una vía, previa transformación de los datos con raíz cuadrada y la prueba a posteriori HSD ('Honestly Significant Difference') de Tukey (Zar 1999). Debido a que los resultados de los análisis de varianza mostraron dos grupos de playas con diferencias significativas en la cantidad de macroalgas varadas (ver Fig. 2), se examinó si esos dos grupos presentaban diferencias significativas en las características físicas. Para esto se realizó el análisis multivariado de Escalamiento Multidimensional no Métrico (EMDNM), el cual se basó en una matriz de similitud calculada mediante la distancia Euclidiana Normalizada (Clarke \& Warwick 1994) y la rutina de análisis de similitudes (ANOSIM) (Clarke \& Warwick 1994) del paquete estadístico PRIMER (Plymouth Routines in Multivariate Ecological Research) (Carr

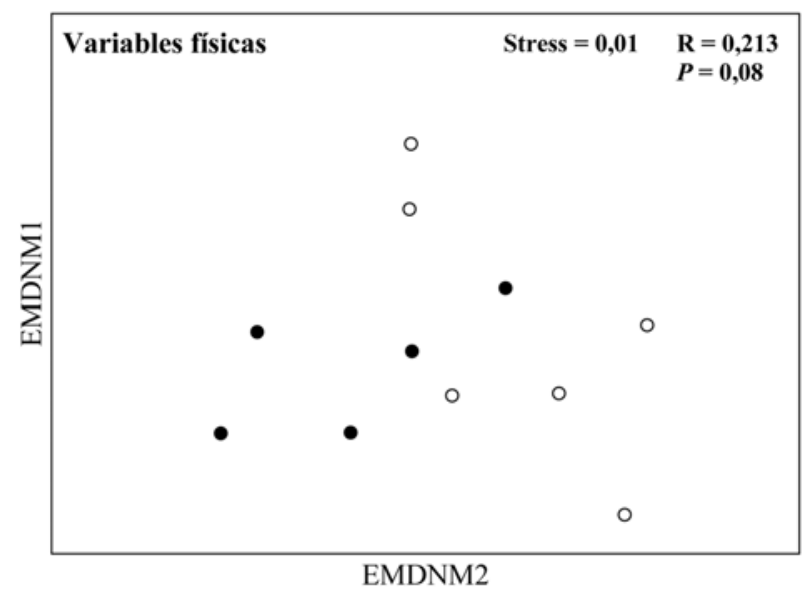

Figura 3

Representación gráfica del Escalamiento Multidimensional no Métrico (EMDNM) obtenida con los valores de las variables físicas (ver Material y métodos para detalles). Valores de $R$ y $\boldsymbol{P}$ son resultantes de la prueba ANOSIM. Los círculos negros corresponden a las playas con los valores más altos de biomasa de macroalgas varadas

Graphic display of the Non Metric Multidimensional Scaling (NMMDS) obtained with the values of physical variables (see Material and methods for details). R and $P$ values are resulting from ANOSIM test. The black circles correspond to the beaches with the highest biomass values of stranded macroalgae

1997). Ya que la prueba de ANOSIM no mostró diferencias significativas $(P>0,05)$ entre las características físicas de las playas que presentaron diferencias significativas en la cantidad de macroalgas varadas (Fig. 3), las playas dentro de cada uno de esos grupos fueron consideradas como réplicas para los análisis comparativos de las abundancias poblacionales y rasgos de historia de vida de $O$. tuberculata. De este modo, desde cada playa se obtuvo un solo valor para cada variable biológica estudiada (e.g. abundancia total, abundancia de adultos), el cual se obtuvo promediando todos los meses y transectos muestreados.

Para comparar los valores de abundancia total, abundancia de cada uno de los componentes de la población (i.e. juveniles, machos, hembras, hembras ovígeras) y del tamaño corporal más grande (i.e. percentil 95) entre los dos grupos de playas, se utilizó análisis de varianza de una vía. Los datos fueron previamente transformados con $\log (\mathrm{x}+1)$ para cumplir con los supuestos de normalidad y homocedasticidad del análisis (Zar 1999). Para comparar la fecundidad de las hembras ovígeras entre las playas que presentaron diferencias en 
la cantidad de macroalgas varadas, se realizó un análisis de covarianza, utilizándose como covariable el tamaño corporal (Zar 1999).

Todos los análisis univariados fueron desarrollados con el programa Statgraphic ${ }^{\circledR}$ v.2.0, en tanto que los multivariados fueron realizados con el programa PRIMER (Carr 1997).

\section{Resultados}

\section{Biomasa de macroalgas varadas}

La biomasa total de macroalgas varadas presentó valores promedios extremos de 0,3 y 7,4 $\mathrm{kg} \mathrm{m}^{-1}$ en las playas de Mehuín y San Ignacio, respectivamente (Fig. 2). Las macrófitas más comunes fueron Durvillaea antarctica (Chamisso), Macrocystis pyrifera Linnaeus y Lessonia nigrescens Bory. Otra de las especies presentes (aunque con menor representatividad y sólo en una playa) fue el alga roja Gigartina skottsbergii Setchell y Gardner. Los resultados de los análisis de varianza llevados a cabo para evaluar las eventuales diferencias entre los valores de biomasa de macroalgas varadas entre las distintas playas se muestran en la Fig. 2. Las playas de Curiñanco Sur, Curiñanco Norte, Mehuín, Universitaria, Pichicullín y
Cheuque no presentaron diferencias significativas $(P>$ $0,05)$ entre sí en la biomasa de macroalgas varadas en las mismas. Esos valores fueron significativamente más bajos $(P<0,05)$ a los registrados en las playas de San Ignacio, La Misión, Calfuco Sur, Calfuco Norte y Caleta Curiñanco, sitios que no presentaron diferencias significativas entre sí $(P>0,05)$ al comparar biomasa de macroalgas varadas en las mismas (Fig. 2).

\section{Características físicas de las playas}

Las playas de Mehuín y Cheuque registraron los sedimentos más finos (266,6 y 249,5 $\mu$ m en promedio, respectivamente), en tanto que Caleta Curiñanco y Curiñanco Norte registraron los sedimentos más gruesos (525,3 y 499,4 $\mu \mathrm{m}$ respectivamente) (Tabla 1$)$. Los valores más altos de altura de la ola se registraron en las playas de Curiñanco Sur y Curiñanco Norte, con valores promedio 324,7 y $347,2 \mathrm{~cm}$, respectivamente, mientras que los más bajos ocurrieron en las playas de La Misión y Caleta Curiñanco $(92,4$ y 72,6 cm, respectivamente) (Tabla 1). Los periodos de ola más largos ocurrieron en Curiñanco Norte y Cheuque (18,3 y 18,8 s, respectivamente), en tanto que en San Ignacio y Caleta Curiñanco ocurrieron los más cortos $(10,4$ y $9,7 \mathrm{~s}$, respectivamente) (Tabla 1 ). Las playas con menor

\section{Tabla 1}

Tamaño medio de la arena, altura y período la ola, pendiente de la zona intermareal (1/p) y parámetro de Dean $(\Omega)$ de las playas estudiadas. Los valores son promedios basados en mediciones mensuales $(n=13)$, realizados en un solo punto de la playa para el tamaño de la arena y características del oleaje y sobre cuatro transectos para el caso de la pendiente (ver Material y métodos para detalles). Desviación estándar en paréntesis

Mean grain size of sands, height and period of waves, slope of the intertidal zone (1/p) and Dean's parameter $(\Omega)$ of the beaches studied. The values are means based on monthly measurements $(n=13)$ carried out in a single point of the beach for mean grain size and wave features and on four transects for the slope of the intertidal zone

(see Material and methods for details). Standard deviation in parentheses

\begin{tabular}{llcccc}
\hline Playas & $\begin{array}{c}\text { Tamaño medio } \\
\text { del grano }(\mu \mathrm{m})\end{array}$ & $\begin{array}{c}\text { Altura de la ola } \\
(\mathrm{cm})\end{array}$ & $\begin{array}{c}\text { Periodo de la ola } \\
(\mathrm{s})\end{array}$ & $\begin{array}{c}\text { Pendiente } \\
1 / \mathrm{p}\end{array}$ & $\Omega$ \\
\hline San Ignacio & $375,7(41,6)$ & $112,6(42,2)$ & $10,4(2,4)$ & 18,1 & $2,1(0,9)$ \\
La Misión & $272,1(18,9)$ & $92,4(45,9)$ & $13,9(2,9)$ & 27,0 & $1,9(1,1)$ \\
Calfuco Sur & $342,3(22,4)$ & $202,1(79,3)$ & $16,7(3,2)$ & 26,8 & $2,5(0,8)$ \\
Calfuco Norte & $370,6(41,3)$ & $309,2(75,1)$ & $17,3(3,0)$ & 35,7 & $3,4(1,2)$ \\
Curiñanco Sur & $346,7(65,9)$ & $324,7(117,8)$ & $17,6(2,4)$ & 21,4 & $2,9(1,1)$ \\
Curiñanco Norte & $499,4(103,1)$ & $347,2(104,8)$ & $18,3(1,8)$ & 21,3 & $2,6(0,7)$ \\
Caleta Curiñanco & $525,3(172,5)$ & $72,6(28,1)$ & $9,7(2,9)$ & 17,9 & $1,0(0,5)$ \\
Mehuín & $266,6(23,3)$ & $269,2(142,1)$ & $17,1(2,3)$ & 34,6 & $4,3(1,7)$ \\
Universitaria & $305,9(26,7)$ & $221,7(75,4)$ & $15,9(2,1)$ & 25,5 & $3,4(1,3)$ \\
Pichicullín & $268,4(41,4)$ & $226,8(100,1)$ & $16,7(1,9)$ & 54,4 & $3,9(1,8)$ \\
Cheuque & $249,5(24,5)$ & $320,2(90,4)$ & $18,8(3,4)$ & 35,7 & $5,3(1,7)$ \\
\hline
\end{tabular}




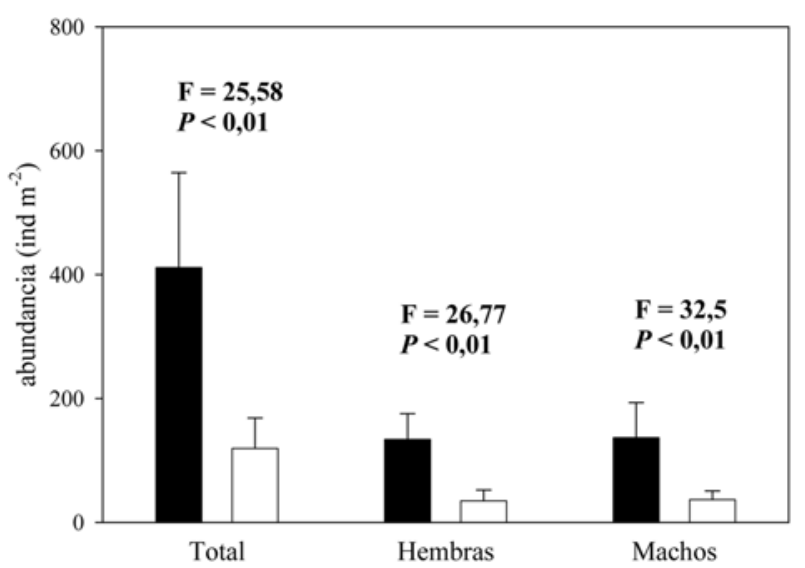

Figura 4

\begin{abstract}
Abundancia total y abundancias de hembras y machos (ind $\mathrm{m}^{-2}$ ) de $O$. tuberculata. Los valores son promedios ( \pm 1 desviación estándar) basados en cuatro transectos muestreados estacionalmente $(n=13)$ (ver Material y métodos para detalles). Valores de $\mathrm{F}$ y $\boldsymbol{P}$ son resultantes

de los análisis de varianza de una vía. Las barras negras representan las playas con los valores más altos de biomasa de macroalgas varadas
\end{abstract}

Total abundance and abundance of females and males (ind $\left.\mathrm{m}^{-2}\right)$ of $O$. tuberculata. The values are means $( \pm 1$ standard deviation) based on the four transects sampled monthly (n = 13) (see Material and methods for details). F and $P$ values are resulting from one-way analyses of variance.

The black bars correspond to the beaches with the

highest biomass values of stranded macroalgae

inclinación fueron las de Pichicullín, Cheuque y Calfuco Norte (54,4 para la primera y 35,7 para las últimas), en tanto que las más inclinadas fueron San Ignacio $(18,1)$ y Caleta Curiñanco $(17,9)$ (Tabla 1). Los valores del parámetro de Dean $(\Omega)$ muestran que todas las playas estudiadas corresponden al tipo de playa intermedia $(\Omega>1<6)$, excepto Caleta Curiñanco, la cual registró características morfodinámicas de tipo reflectivas $(\Omega \leq$ 1) (Tabla 1).

\section{Abundancias totales y rasgos de historia de vida de Orchestoidea tuberculata}

Las abundancias poblacionales totales, de machos y hembras de $O$. tuberculata, fueron significativamente más altas $(P<0,05)$ en las playas que presentaron los valores significativamente más altos de macroalgas varadas (Fig. 4). Resultados similares se registraron para la abundancia de hembras ovígeras, como también para la abundancia de los juveniles, los cuales se consideran una medida directa del éxito en el reclutamiento (Fig. 5). El tamaño

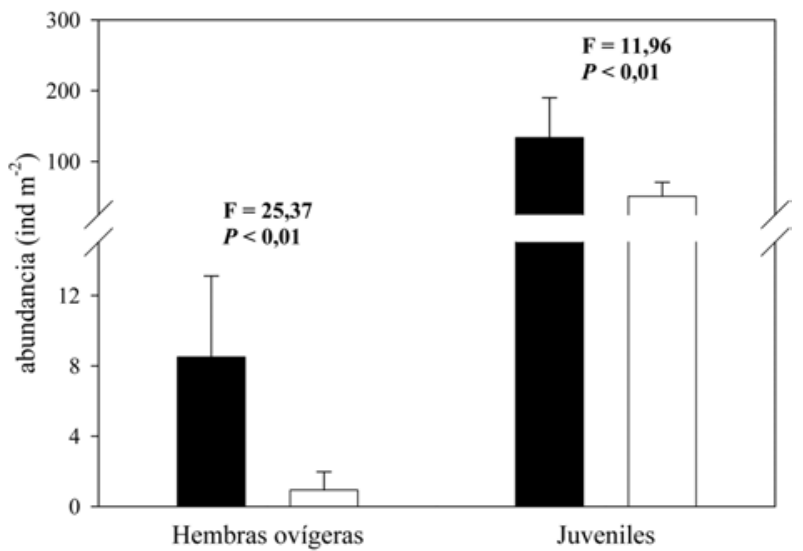

Figura 5

Abundancia (ind $\mathbf{m}^{-2}$ ) de hembras ovígeras y juveniles de $O$. tuberculata. Los valores son promedios $( \pm 1$ desviación estándar) basados en cuatro transectos muestreados estacionalmente $(n=13)$ (ver Material y métodos para detalles). Valores de $\mathbf{F}$ y $\boldsymbol{P}$ son resultantes de los análisis de varianza de una vía. Las barras negras representan las playas con los valores más altos de biomasa de macroalgas varadas

Abundance (ind $\mathrm{m}^{-2}$ ) of ovigerous females and juveniles of $O$. tuberculata. The values are means $( \pm 1$ standard deviation) based on the four transects sampled monthly (n $=13$ ) (see Material and methods for details). F and $P$ values are resulting from one-way analyses of variance.

The black bars correspond to the beaches with the highest biomass values of stranded macroalgae

corporal de los ejemplares de $O$. tuberculata fue significativamente más alto $(P<0,05)$ en las playas con valores más altos de macroalgas varadas; i.e. tamaños corporales con aproximadamente $2 \mathrm{~mm}$ más de longitud que aquellos de las playas con menores cantidades de macroalgas varadas (Fig. 6). La fecundidad, no presentó diferencias significativas $(P>0,05)$ entre playas con diferentes cantidades de macroalgas varadas (Fig. 7). Sin embargo, el número esperado de huevos por hembra ovígera (estimado mediante la multiplicación del número promedio de hembras ovígeras por la fecundidad promedio individual), fue nueve veces más alto en las playas con mayor cantidad de macroalgas varadas.

\section{Discusión}

Los resultados de este estudio sugieren que los subsidios de las macroalgas influyen significativamente sobre las poblaciones de $O$. tuberculata en playas arenosas de la costa del centro sur de Chile. Los valores de la abundancia total, abundancia de hembras, hembras ovígeras, machos 


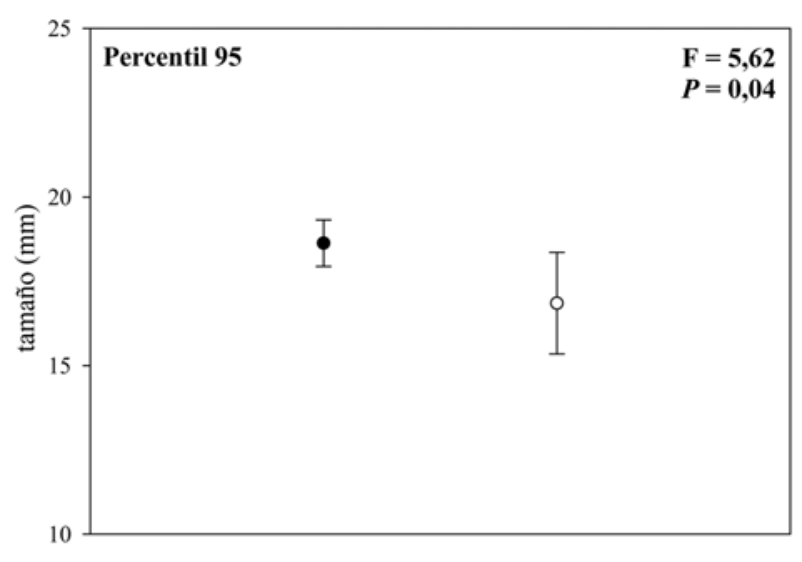

Figura 6

Percentil 95 del tamaño corporal de $O$. tuberculata. Los valores son promedios ( \pm 1 desviación estándar) basados en cuatro transectos muestreados estacionalmente ( $n=13)$ (ver Material y métodos para detalles). Valores de $F$ y $P$ son resultantes del análisis de varianza de una vía. Los círculos negros representan las playas con los valores más altos de biomasa de macroalgas varadas

$95^{\text {th }}$ percentile of the body size of $O$. tuberculata. The values are means $( \pm 1$ standard deviation) based on the four transects sampled monthly $(\mathrm{n}=13)$ (see Material and methods for details). $\mathrm{F}$ and $P$ values are resulting from one way analysis of variance. The black circles correspond to the beaches with the highest biomass values of stranded macroalgae

y juveniles, además del tamaño corporal, fueron significativamente más altos en playas con mayor cantidad de macroalgas varadas. Las playas comparadas (i.e. aquellas con diferencias en la cantidad de macroalgas varadas), no presentaron diferencias significativas en las características físicas, lo que permite sugerir que las características físicas y estado morfodinámico de las playas estudiadas no influyen significativamente sobre las poblaciones de $O$. tuberculata en el área de estudio. Por lo tanto, el patrón observado en este estudio no concuerda con las predicciones de la hipótesis de rigurosidad del hábitat (Defeo et al. 2003) y las de la hipótesis de protección del hábitat (Defeo \& Gómez 2005), las cuales predicen un incremento (la primera) o una disminución (la segunda) de los atributos poblacionales (e.g. abundancia total, abundancia de juveniles), desde playas reflectivas hacia playas intermedias y disipativas.

En muchos sistemas costeros, el subsidio de macrófitas aumenta la producción de consumidores

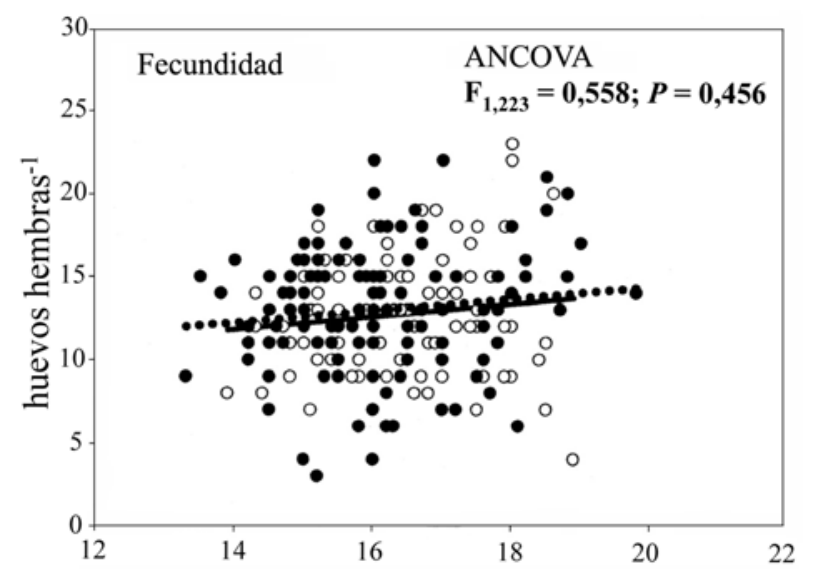

Figura 7

Fecundidad $v$ s. tamaño corporal de $O$. tuberculata. Valores de F y $\boldsymbol{P}$ son resultantes del análisis de covarianza. Los círculos de igual color representan playas que no presentaron diferencias significativas en los valores de biomasa de macroalgas varadas, correspondiendo los de color negro a las playas con los valores más altos de biomasa

Fecundity $v s$. body size of $O$. tuberculata. $\mathrm{F}$ and $P$ values are resulting from analysis of covariance. Circles with the same colour represent beaches without significant differences in their biomass of stranded macroalgae; black circles correspond to the beaches with the highest biomass values

primarios y secundarios (Bustamante \& Branch 1996, Bustamante et al. 1995, Duggins et al. 1989, Menge 1992, Vetter \& Dayton 1999, Rodríguez 2000, 2003), lo que también ha sido observado en islas, donde incluso los subsidios marinos superan la productividad in situ (Polis \& Hurd 1995, Anderson \& Polis 1998), situación que influye consecuentemente en la organización de las poblaciones y comunidades costeras. Por ejemplo, en la costa rocosa de Sudáfrica, Bustamante et al. (1995) registraron que el subsidio de macroalgas es clave para mantener las altas densidades de los dos gasterópodos numéricamente dominantes (Patella argenvillei Krauss y Patella granatina Linnaeus), las cuales a su vez ejercen un significativo efecto 'top-down' lo que favorece indirectamente el desarrollo de algas coralinas crustosas e inhibe la invasión del substrato primario por especies de invertebrados filtradores. En la costa central de Chile (ca. $33^{\circ} \mathrm{S}$ ), Rodríguez (2003) mostró que la principal fuente alimentaria del erizo Tetrapygus niger (Molina) son macroalgas a la deriva depositadas sobre el intermareal rocoso, lo que resulta en una menor presión de pastoreo por parte de este herbívoro sobre las algas del área. Estos resultados llevaron a este autor a proponer que el consumo de estos subsidios, podía tener 
consecuencias significativas a nivel comunitario en esta área de la costa de Chile. Aún cuando estudios previos llevados a cabo en playas arenosas dan sustento a la hipótesis de que los subsidios de macroalgas pueden influir sobre la macrofauna en estos ambientes (e.g. Griffiths et al. 1983, Dugan et al. 2003), estos han estado basados en muestreos puntuales o solo en una sola playa. Consecuentemente, el presente estudio es el primero en cuantificar el rol de los subsidios de macroalgas sobre los atributos poblacionales de un componente importante de la macrofauna, en un rango morfodinámico de playas arenosas y sobre la base de muestreos estaciónales.

La estimación de la cantidad de macroalgas varadas realizada en este estudio, representa una medición puntual del arribo mensual y no la cantidad real que llega a la playa o la tasa de recambio de las mismas. Varios factores complican este tipo de mediciones. En primer lugar, la depositación de este subsidio es un proceso extremadamente dinámico, tanto en el espacio como en el tiempo (Griffiths et al. 1983, Rodríguez 2003, Orr et al. 2005), con eventos frecuentes de suspensión y redepositación durante los distintos ciclos mareales (Colombini et al. 2000, Orr et al. 2005). Por otra parte, una cantidad importante de macroalgas puede ser consumida diariamente por organismos detritívoros. Por ejemplo, Dugan et al. (2003) señalan que cuando la cantidad de macroalgas varadas es escasa, estas pueden ser consumidas totalmente durante un ciclo mareal. A pesar de lo anterior, las estimaciones realizadas en este estudio (sobre la base de 13 meses de muestreo) permiten asumir razonablemente, que aquellas playas donde se registraron los valores promedios más altos de biomasa de macroalgas varadas, son las que reciben las mayores cantidades de este tipo de subsidio trófico.

La cantidad de macroalgas que arriban a las playas arenosas puede estar afectada por muchos factores. La diferencia en la cantidad de macroalgas identificada en dos grupos de playas aquí estudiadas (i.e. playas con alta cantidad y con baja cantidad de macroalgas varadas), puede estar relacionada en parte a la disponibilidad de macroalgas en las costas rocosas adyacentes, las cuales son una de las principales fuentes de estos subsidios tróficos. Esta disponibilidad puede estar afectada por factores tales como sobrepastoreo de herbívoros, eventos ENSO (El Niño-Oscilación del sur) del o sobre explotación realizada por el hombre, la cual es significativa en esta área del litoral chileno (Moreno 2001). Por ejemplo, cuatro de las seis playas que registraron los valores más bajos de macroalgas varadas (i.e. Mehuín, Universitaria, Pichicullín y Cheuque), se ubican en una zona de muy fácil acceso y donde se observó una importante actividad humana asociada a la extracción de macrófitas desde las zonas rocosas aledañas. Observaciones directas de esas zonas rocosas, muestran una cobertura muy baja de macroalgas, lo que explicaría las bajas cantidades de este tipo de subsidios en la zona intermareal de esas playas. Estos resultados sugieren que la remoción de macroalgas desde zonas rocosas aledañas a playas arenosas, puede cambiar a mesoescala la estructura poblacional de $O$. tuberculata (ver Castilla 1999, Moreno 2001, para ejemplos en costas rocosas). Para otras áreas litorales de Chile y el mundo se ha demostrado que factores tales como cercanía a la fuente de macroalgas y procesos hidrológicos locales (e.g. oleaje), también pueden influir sobre la exportación de macroalgas a otros sistemas. Por ejemplo, en la costa central de Chile ( $c a .33^{\circ} \mathrm{S}$ ), Rodríguez (2003) mostró que la mayor cantidad de macroalgas varadas ocurren en sectores con presencia de bosques de macroalgas y con suave acción del oleaje. Similarmente, Dugan et al. (2003), mostraron en la costa de California, que la cercanía con la fuente de macroalgas fue uno de los factores que influyó sobre la cantidad de algas que arriban a las playas del área, a la vez que Ince et al. (2007) sugirieron que las diferencias en los procesos hidrológicos locales explicarían las diferencias en la cantidad de macroalgas varadas que arriban a las playas del sur de Australia.

Los estudios desarrollados para evaluar las respuestas poblacionales de los organismos que habitan en los niveles superiores de la zona intermareal vs. estados morfodinámicos de playas arenosas, han mostrado resultados altamente disímiles. Por ejemplo, Defeo \& Gómez (2005) encontraron que en playas de la costa de Uruguay, el anfípodo Atlantorchestoidea brasiliensis (Dana) muestra un incremento en sus abundancias totales, abundancias de hembras ovígeras y juveniles, así como también en tamaños corporal, desde playas disipativas a playas reflectivas. Resultados similares fueron registrados en la costa centro sur de Chile por Contreras et al. (2003), quienes mostraron que las abundancias totales más bajas (así como las de hembras ovígeras y juveniles) y menores tasas de crecimiento de $O$. tuberculata, ocurrieron en una playa con características morfodinámicas próximas al tipo disipativo (Mehuín). En contraste a lo anterior, Jaramillo \& McLachlan (1993), mostraron que O. tuberculata y el isópodo cirolánido Excirolana brasiliensis Richardson, incrementaron sus abundancias hacia playas con características disipativas. Abundancias más altas hacia el extremo disipativo, fueron también registradas por Jaramillo et al. (2000) para ese anfípodo en playas arenosas de la costa expuesta de la Isla de Chiloé ( $\mathrm{ca}$. $40^{\circ} \mathrm{S}$ ). Los resultados de este estudio, sugieren que la inconsistencia en la relación atributos poblacionales de 
los organismos que habitan en los niveles superiores de la playa y el estado morfodinámico de las mismas, puede ser explicado por la influencia de la disponibilidad de macroalgas varadas u otro tipo de subsidio tróficos, aspecto no considerado en ninguno de los trabajos mencionados anteriormente. Contreras et al. (2003) hipotetizaron que las diferencias en las abundancias poblacionales totales, de hembras ovígeras y juveniles de $O$. tuberculata mencionadas anteriormente, podrían estar relacionadas con la mayor disponibilidad de macroalgas varadas observadas en la playa intermedia de Calfuco Norte vs. la de Mehuín. Los resultados de la presente investigación, en la cual se incluyeron esas dos playas, confirman las predicciones de la hipótesis presentada por Contreras et al. (2003) debido a que: i) los valores más altos de macroalgas varadas se registraron en la playa de Calfuco (ver Fig. 2) y ii) en las playas con mayor cantidad de macroalgas, se registraron los valores más altos de los atributos poblacionales estimados para O. tuberculata. Siguiendo el mismo razonamiento anterior, es posible asumir que el crecimiento más rápido registrado para $O$. tuberculata en la playa de Calfuco Norte, también sería el resultado de la mayor disponibilidad de macroalgas en esa playa comparada con la de Mehuín (ver Contreras et al. 2003), una hipótesis que también ha sido propuesta por Marques et al. (2003) para explicar el mayor crecimiento del anfípodo Talitrus saltator en las playas arenosas de Lavos (Portugal) y Collelungo (Italia) comparado con el crecimiento por este talítrido en la playa de Zouara (Túnez).

La fecundidad fue el único parámetro poblacional que no presentó diferencias significativas entre playas con diferencias en la cantidad de macroalgas varadas. Esta característica reproductiva puede estar significativamente influenciada, entre otros factores, por la disponibilidad y calidad del alimento (Koop \& Field 1980, Cruz-Rivera \& Hay 2000), situación que no fue evaluada en este estudio así como la viabilidad de los huevos, la cual puede ser afectada por la disponibilidad y calidad de alimentos, sin que necesariamente se produzca una reducción en el número de los mismos (e.g. Chaudron et al. 1996). Sin embargo, cuando se consideró el número esperado de huevos por hembra ovígera, las playas con mayor cantidad de macroalgas varadas presentaron valores nueve veces más altos que aquellas con menor cantidad, lo que es consistente con las mayores abundancias de juveniles registradas en esas playas.

En conclusión, los resultados obtenidos en este estudio (ver también Dugan et al. 2003), sugieren que el cuerpo de ideas desarrollado en ecología de playas arenosas en relación a la influencia de las características físicas de estas playas sobre la macrofauna, debe ser refinado, principalmente para las especies que ocurren en los niveles superiores de la zona intermareal. Por lo tanto, estudios futuros conducentes a entender los procesos que estructuran las comunidades y poblaciones en playas arenosas, deberán considerar el rol del subsidio de macroalgas, tomando en cuenta la composición específica de las mismas, ya que estudios recientes han mostrado que $O$. tuberculata y otros anfípodos talítridos, presentan preferencias alimentarias entre las distintas algas disponibles (Duarte et al. 2008, Lastra et al. 2008) y que sus mayores tasas de crecimiento ocurren al consumir las algas preferidas (Lastra et al. 2008).

\section{Agradecimientos}

Este estudio fue financiado por la Dirección de Investigación y Desarrollo de la Universidad Austral de Chile (Proyecto D-2003-15 a CD) y CONICYT-CHILE (Proyecto FONDECYT no 3085005 a CD). Los autores agradecen el apoyo en terreno de Erwin Barría. Los comentarios anónimos de los evaluadores de este trabajo contribuyeron de manera significativa al mejoramiento del mismo.

\section{Literatura citada}

Anderson WB \& GA Polis. 1998. Marine subsidies of island communities in the Gulf of California: evidence from stable carbon and nitrogen isotopes. Oikos 81: 75-80.

Bascom W. 1980. Waves and beaches, 366 pp. Anchor Press, New York.

Brown A \& A McLachlan. 1990. Ecology of sandy shores, 328 pp. Elsevier Science Publishers, Amsterdam.

Bustamante RH \& GM Branch. 1996. The dependence of intertidal consumers on kelp-derived organic matter on the west coast of South Africa. Journal of Experimental Marine Biology and Ecology 196: 1-28.

Bustamante RH, GM Branch \& S Aekhout. 1995. Maintenance of an exceptional grazer biomass on South African intertidal shores: trophic subsidy by subtidal kelps. Ecology 76: 2314-2329.

Carr MR. 1997. Primer user manual, 40 pp. Plymouth Marine Laboratory, Plymouth.

Castilla JC. 1999. Coastal marine communities: trends and perspectjves from human-exclusion experiments. Trends in Ecology \& Evolution 14: 280-283.

Chaudron Y, SA Poulet, M Laabir, A Lanora \& A Miralto. 1996. Is hatching success of copepod eggs diatom densitydependent? Marine Ecology Progress Series 144: 185-193.

Clarke KR \& RM Warwick. 1994. Change in marine communities: An approach to statistical analysis and interpretation, 144 pp. Plymouth Marine Laboratory, Plymouth.

Colombini I, A Aloia, M Fallaci, G Pezzoli \& L Chelazzi. 2000. Temporal and spatial use of stranded wrack by the 
macrofauna of a tropical sandy beach. Marine Biology 136: 531-541.

Contreras H, E Jaramillo, C Duarte \& A McLachlan. 2003. Population abundances, growth and natural mortality of the crustacean macrofauna at two sand beach morphodynamic types in southem Chile. Revista Chilena de Historia Natural 76: 543-561.

Cruz-Rivera E \& MH Hay. 2000. The effects of diet mixing on consumer fitness: macroalgae, epiphytes, and animal matters as food for marine amphipods. Oecologia 123: 252264.

Defeo 0 \& J Gómez. 2005. Morphodynamics and habitat safety in sandy beaches: life-history adaptations in a supralittoral amphipod. Marine Ecology Progress Series 293: 143-153.

Defeo $O$ \& M Rueda. 2002. Spatial structure, sampling design and abundance estimates in sandy beach macroinfauna: some warnings and new perspectives. Marine Biology 140: $1215-1225$

Defeo O, A Brazeiro, A De Alava \& G Riostra. 1997. Is sandy beach macrofauna only physically controlled? Role of substrate and competition in isopods. Estuarine, Coastal and Shelf Science 45: 453-462.

Defeo O, D Lercari \& J Gómez. 2003. The role of morphodynamics in structuring sandy beach populations and communities: what should be expected? Journal of Coastal Research (Special Issue) 35: 352-362.

Duarte W. 1974. Orchestoidea tuberculata Nicolet, 1849 como organismo desintegrador de algas (Crustacea, Amphipoda, Talitridae). Nota Mensual Museo Nacional de Historia Natural (Chile) 220-221: 3-9.

Duarte C, E Jaramillo \& H Contreras. 2008. Macroalgas varadas sobre la superficie de una playa arenosa del sur de Chile: preferencias alimentarias y de hábitat de juveniles y adultos de Orchestoidea tuberculata (Nicolet), (Amphipoda, Talitridae). Revista Chilena de Historia Natural 80: 69-81.

Dugan J, DM Hubbard, MD McCrary \& MO Pierson. 2003. The response of macrofauna communities and shorebirds to macrophyte wrack subsidies on exposed sandy beach of southern California. Estuarine, Coastal and Shelf Science 58: $25-40$

Duggins DO, CA Simenstad \& JA Estes. 1989. Magnification of secondary production by kelp detritus in coastal marine ecosystems. Science 41: 170-173.

Emery KO. 1938. Rapid method of mechanical analysis of sands. Journal of Sedimentary Petrology 8: 105-111.

Emery KO. 1961. A simple method of measuring beach profiles. Limnology and Oceanography 6: 90-93.

Fairweather PG \& GP Quinn. 1992. Seascape ecology: the importance of linkages. En: Battershill CN (ed). Proceedings of the Second International Temperate Reef Symposium, 7-10 January, 1992, pp. 77-83, NIWA Marine, Auckland.
Gibbs RJ, MD Mathews \& DA Link. 1971. The relationship between sphere size and settling velocity. Journal of Sedimentary Petrology 41: 7-18.

Griffiths CL \& J Stenton-Dozey. 1981. The fauna and rate of degradation of stranded kelp. Estuarine, Coastal and Shelf Science 12: 645-653.

Griffiths CL, J Stenton-Dozey \& K Koop. 1983. Kelp wrack and the flow of energy through a sandy beach ecosystem. En: McLachlan A \& T Erasmus (eds). Sandy beaches as ecosystems, pp. 547-556. Dr. W Junk Publishers, The Hague.

Ince RT, GA Hyndes, PS Lavery \& MA Vanderklift. 2007. Marine macrophytes directly enhance abundances of sandy beach fauna through provision of food and habitat. Estuarine, Coastal and Shelf Science 74: 77-86.

Inglis G. 1989. The colonisation and degradation of stranded Macrocystis pyrifera (L) C. Ag. by the macrofauna of a New Zealand sandy beach. Journal of Experimental Marine Biology and Ecology 125: 203-217.

Jaramillo E. 1987. Community ecology of Chilean sandy beaches. Ph.D. Dissertation, University of New Hampshire, Durham, 216 pp.

Jaramillo E \& A McLachlan. 1993. Community and population responses of the macrofauna to physical factors over a range of exposed sandy beaches in south-central Chile. Estuarine, Coastal and Shelf Science 37: 615-624.

Jaramillo E, C Duarte \& H Contreras. 2000. Sandy beach macrofauna from the coast of Ancud, Isla de Chiloé, southern Chile. Revista Chilena de Historia Natural 73: 771-786.

Jaramillo E, H Contreras, C Duarte \& P Quijón. 2001. Relationships between community structure of the intertidal macrofauna and sandy beach characteristics along the Chilean Coast. Marine Ecology 22: 323-342.

Jaramillo E, H Contreras, C Duarte \& MH Avellanal. 2003. Locomotor activity and zonation of upper shore arthropods in a sandy beach of north central Chile. Estuarine, Coastal and Shelf Science 58: 177-197.

Jaramillo E, W Stotz, C Bertran, J Navarro, C Roman \& C Vareta. 1980. Actividad locomotríz de Orchestoidea tuberculata (Amphipoda, Talitridae) sobre la superficie de una playa arenosa del sur de Chile (Mehuín, Valdivia). Studies on Neotropical Fauna and Environment 15: 9-33.

Koop K \& JG Field. 1980. The influence of food availability on population dynamics of a supralittoral isopod Ligia dilatata Brand. Journal of Experimental Marine Biology and Ecology 48: 61-72.

Lastra M, HM Page, J Dugan, DM Hubbard \& F Rodil. 2008. Processing of allochthonous macrophyte subsidies by sandy beach consumer: estimates of feeding rates and impacts on food resources. Marine Biology 154: 163-174.

Lewin R. 1986. Supply-side ecology. Science 234: 25-27. 
Marques JC, SC Gonçalves, MA Pardal, L Chelazzi, I Colombini, M Fallaci, MF Bouslama, M El Gtari, F Charfi-Cheikhruha \& F Sacapini. 2003. Comparison of Talitrus saltator (Amphipoda, Talitridae) biology, dynamics, and secondary production in Atlantic (Portugal) and Mediterranean (Italy and Tunisia) populations. Estuarine, Coastal and Shelf Science 58 (Suppl. 1): 127148.

McArdle S \& A McLachlan. 1991. Dynamics of the swash zone and effluent line on sandy beaches. Marine Ecology Progress Series 76: 91-99.

McArdle S \& A McLachlan. 1992. Sandy beach ecology: swash features relevant to the macrofauna. Journal of Coastal Research 8: 398-407.

McLachlan A. 1990. Dissipative beaches and macrofauna communities on exposed intertidal sands. Journal of Coastal Research 6: 57-71.

McLachlan A \& E Jaramillo. 1995. Zonation on sandy beaches. Oceanography and Marine Biology: an Annual Review 33: 305-335.

McLachlan A, A De Ruyck \& N Hacking. 1996. Community structure on sandy beaches: patterns of richness and zonation in relation to tide range and latitude. Revista Chilena de Historia Natural 69: 451-467.

McLachlan A, E Jaramillo, TE Donn \& F Wessels. 1993. Sand beach macrofauna communities: a geographical comparison. Journal of Coastal Research, (Special Issue) 15: $27-38$

McLachlan A, M Fisher, H Al-Habsi, S Al-Shukairi \& AAlHabsi. 1998. Ecology of sandy beaches in Oman. Journal of Coastal Conservation 4: 181-190.

Menge BA. 1992. Community regulation: under what conditions are bottom-up factors important on rocky shores? Ecology 73: 755-765.

Moreno C. 2001. Community patterns generated by human harvesting on Chilean shores: a review. Aquatic Conservation: Marine and Freshwater Ecosystems 11: 1930 .

Nielsen KJ \& SA Navarrete. 2004. Mesoscale regulation comes from the bottom-up: intertidal interactions between consumers and upwelling. Ecology Letters 7: 31-41

Orr M, M Zimmer, D Jelinski \& M Mews. 2005. Wrack deposition on different beach types: spatial and temporal variation in the pattern of subsidy. Ecology 86: 1496-1507.

Polis GA \& SD Hurd. 1995. Extraordinarily high spider densities on islands: flow of energy from the marine to terrestrial food webs and the absence of predation.
Proceedings from the National Academy of Science of the United States 92: 4382-4386.

Polis GA, WB Anderson \& RD Holt. 1997. Toward an integration of landscape and food web ecology: the dynamics of spatially subsidized food webs. Annual Review of Ecology and Systematic 28: 289-316.

Polis GA, RD Holt, BA Menge \& KO Winemiller. 1995. Time, space, and life history: influences on food webs. En: Polis GA \& K Winemiller (eds). Food webs: integration of patterns and dynamics, pp. 435-460, Chapman \& Hall, London.

Rodríguez S. 2000. Transferencia de recursos alimentarios entre diferentes ambientes del ecosistema marino. Revista Chilena de Historia Natural 73: 199-207.

Rodríguez S. 2003. Consumption of drift kelp by intertidal populations of the sea urchin Tetrapygus niger on the central Chilean coast: possible consequences at different ecological levels. Marine Ecology Progress Series 251: 141-151.

Rossi F \& AJ Underwood. 2002. Small-scale disturbance and increased nutrients as influences on intertidal macrobenthic assemblages: experimental burial of wrack in different intertidal environments. Marine Ecology Progress Series 241: 29-39.

Schoeman DS \& AJ Richardson. 2002. Investigating biotic and abiotic factors affecting the recruitment of an intertidal clam on an exposed sandy beach using a generalized additive model. Journal of Experimental Marine Biology and Ecology 276: 67-81.

Seward-Thompson B \& J Hails. 1973. An appraisal on the computation of statistical parameters in grain size analysis. Sedimentology 11: 83-98.

Short A. 1996. The role of wave height, period, slope, tide range and embaymentisation in beach classifications: a review. Revista Chilena de Historia Natural 69: 589-604.

Short A. 2006. Australian beach system-nature and distribution. Journal of Coastal Research 22: 11-27.

Varela C. 1983. Anfípodos de las playas del arena del sur de Chile (Bahía de Maiquillahue, Valdivia). Studies on Neotropical Fauna and Environment 18: 25-52.

Vetter EW \& PK Dayton. 1999. Organic enrichment by macrophyte detritus, and abundance patterns of megafaunal populations in submarine canyons. Marine Ecology Progress Series 186: 137-148

Zar H. 1999. Biostatistical analysis, 662 pp. Prentice-Hall, Upper Saddle River.

Recibido el 21 de abril de 2009 y aceptado el 22 de septiembre de 2009 